
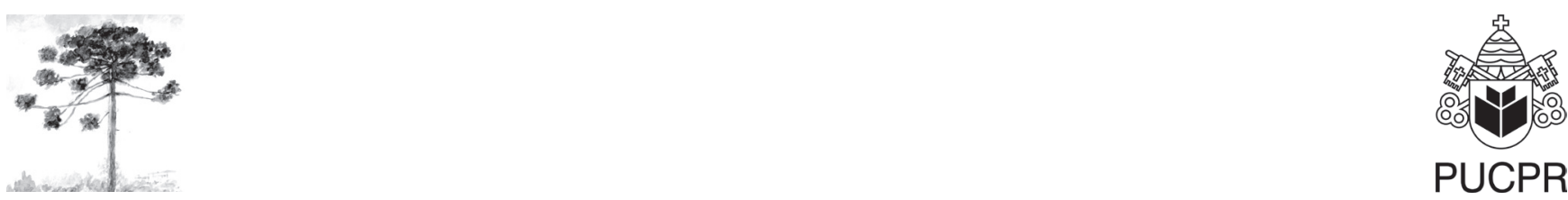

PUCPR

\title{
ASPECTOS CLÍNICOS E MECANISMO DE AÇÃO DAS PRINCIPAIS CLASSES FARMACOLÓGICAS USADAS NO TRATAMENTO DA HIPERTENSÃO ARTERIAL SISTÊMICA
}

\author{
Clinical aspects and action mechanism of main \\ pharmacological classes used in the treatment of bypertension
}

\author{
Anderson Martelli ${ }^{[a]}$, Marco Aurélio Tosta Longo ${ }^{[b]}$, Cleber Seriani ${ }^{[c]}$ \\ [a] Graduado em Ciências Biológicas pela Universidade de Pinhal, Instituto de Ciências Biológicas, Unipinhal, especialização em \\ Laboratório Clínico pela Faculdade de Ciências Médicas da Universidade Estadual de Campinas (Unicamp), Campinas, SP - Brasil, \\ e-mail:martellibio@hotmail.com \\ [b] Graduado em Enfermagem pela Universidade de Pinhal (Unipinhal), Espírito Santo do Pinhal, SP - Brasil. \\ [c] Graduando em Ciências Biológicas pela Universidade de Pinhal (Unipinhal), Instituto de Ciências Biológicas, Espírito Santo do \\ Pinhal, SP - Brasil.
}

\section{Resumo}

A Hipertensão Arterial Sistêmica (HAS) é uma situação clínica de natureza multifatorial caracterizada por níveis de pressão arterial (PA) altos, apresentando uma elevada prevalência na população mundial e no Brasil. Atinge cerca de $20 \%$ da população dos países do mundo ocidental e apesar dos inúmeros avanços no tratamento, apenas a minoria dos pacientes hipertensos apresentam níveis de pressão arterial controlados. A prevalência da HAS aumenta consideravelmente com a idade, multiplicando o risco de danos cardiovasculares, contribuindo para o aumento da morbidade/mortalidade e os custos sociais com a invalidez e absenteísmo ao trabalho. Os estudos nesse campo permitem identificar a HAS como um importante problema de saúde pública. Quanto ao tratamento, os protocolos atuais recomendam principalmente modificações no estilo de vida geralmente associado ao tratamento farmacológico com anti-hipertensivos. O presente trabalho apresenta como objetivo a descrição dos mecanismos de ação das principais classes de fármacos anti-hipertensivos, servindo como modelo de estudo nos quadros caracterizados como HAS.

Palavras-chave: Hipertensão arterial sistêmica. Cardiovascular. Mecanismos de ação. Anti-hipertensivos. 


\begin{abstract}
The Systemic Arterial Hypertension (SAH) is a multifactorial clinical situation characterized by high levels of blood pressure (BP), showing a high prevalence in the world population and in Brazil. Reaches about $20 \%$ of the population of Western countries in the world and, despite many advances in the treatment, only a minority of hypertensive patients have controlled levels of blood pressure. The prevalence of SAH had significantly increased with age, increasing the risk of cardiovascular damage, contributing to increase morbidity/mortality and social costs, resulting to the disability and absenteeism at work. Studies in this field allow identifying the SAH as an important public health problem. For treatment, the current protocols recommend specially changes in lifestyle often associated with pharmacological treatment with anti-hypertensive. The present act has the intention to show a description of the action mechanisms of principal classes of antihypertensive drugs, like a model for study in the background as $S A H$.
\end{abstract}

Keywords: Systemic high blood pressure. Cardiovascular. Mechanisms of action. Anti-hypertensive.

\section{INTRODUÇÃO}

A Hipertensão Arterial Sistêmica (HAS) é uma situação clínica de natureza multifatorial caracterizada por níveis de pressão arterial (PA) elevados. Apresenta alta prevalência na população mundial e no Brasil, com estimativa de que cerca de $15 \%$ dos indivíduos adultos podem ser considerados hipertensos (1).

Apesar dos inúmeros avanços no tratamento, apenas a minoria dos pacientes hipertensos apresentam níveis de pressão arterial controlado (2). A prevalência da HAS aumenta com aidade, multiplicando o risco de danos cardiovasculares, contribuindo para o aumento da morbimortalidade e os custos sociais com invalidez e absenteísmo ao trabalho. O controle adequado dessa situação paradoxalmente reduz os riscos individuais e os custos sociais (1).

Cerca de $90 \%$ a $95 \%$ de todos os casos de hipertensão são ditos de "hipertensão primária", também conhecida como hipertensão arterial essencial, que se apresenta sem uma causa definida $(1,3)$. Em contraste, todas as demais formas de hipertensão, chamadas secundárias, estão associadas a outras patologias ou formas conhecidas e compreendidas $(1,3)$.

Assim, secundariamente temos a obesidade, envelhecimento, diabetes melito, insuficiência renal crônica como importantes fatores associados à dificuldade de controlar a pressão arterial (4), podendo paralelamente fazer parte desse grupo um conjunto de fatores de risco associado ao metabolismo dos lípides (2).

A HAS causa, com o decorrer do tempo, patologias graves (1), mas dados provenientes de alguns estudos sugerem que de $30 \%$ a $50 \%$ dos pacientes hipertensos, mesmo em tratamento medicamentoso, apresentam pressão arterial elevada (4). Esses estudos permitem identificar a HAS como um importante problema de saúde pública, conferindo um alto risco cardiovascular ao paciente $(2,4)$.

Novos estudos e protocolos clínicos sugerem também que a hipertensão arterial primária apresenta uma forte tendência hereditária (3), mostrando que cerca da metade dos pacientes hipertensos correlacionam um traço familiar para hipertensão ou mortalidade cardiovascular prematura em seus parentes de primeiro grau (1).

A maior parte dos pacientes com hipertensão apresenta excesso de peso, e estudos de diferentes populações sugerem que o sobrepeso e a obesidade podem ser responsáveis por entre $65 \%$ e $70 \%$ do risco de desenvolvimento de hipertensão arterial (3).

Estudos clínicos no tratamento da pressão arterial se fazem com modificações do estilo de vida, com a realização de atividades físicas e redução do peso corporal, como primeiro passo na redução da pressão arterial (3), bem como restrição do sal na dieta, associado ou não ao uso de medicamentos, que podem também ser administrados isolados ou em associação $(5,6)$.

Nesse contexto, o estudo retrata os aspectos clínicos da hipertensão arterial, assim como os mecanismos de ação dos fármacos utilizados em seus respectivos tratamentos. Sabendo que a HAS é considerada uma patologia multifatorial que causa considerável número de mortes e com uma alta prevalência na população, um diagnóstico correto e tratamento apropriado podem melhorar 
consideravelmente a sobrevida desses pacientes, levando ao declínio dos índices de mortalidade em comparação com os números que vem sendo observado.

O presente trabalho faz uma revisão de literatura dos aspectos clínicos da HAS com uma abordagem relevante dos mecanismos de ação das principais classes farmacológicas utilizados no tratamento e/ou controle da HAS.

\section{Mecanismos de ação das principais classes de medicamentos anti-hipertensivos}

Diversas classes de anti-hipertensivos já demonstraram reduzir o risco cardiovascular e, na maioria dos casos, é necessário associar fármacos com mecanismos de ação diferentes. Além da evidência de benefício clínico, a escolha do anti-hipertensivo deve considerar diversos fatores, como as comorbidades do paciente, o perfil de efeitos adversos, interação medicamentosa, posologia e até mesmo o preço do fármaco no mercado (7).

Diferentemente dos jovens, os idosos possuem em média três a cinco doenças crônicas e somente $6 \%$ se considera livre de doenças. Assim, as comorbidades frequentemente norteiam a escolha do anti-hipertensivo, devendo dar preferência a fármacos que também possam trazer benefícios às outras doenças existentes (7).

Nos idosos deve-se também avaliar as particularidades no tratamento medicamentoso relativo às alterações fisiológicas próprias do envelhecimento, como diminuição da atividade dos barorreceptores, alterações da composição corpórea, metabolismo basal, fluxo sanguineo hepático, ritmo de filtração glomerular, com alteração de absorção, distribuição e metabolização dos medicamentos (8). A introdução do anti-hipertensivo deve ser feita em doses baixas com um aumento gradual, porém sem perder de vista o alvo de PA desejado. Os pacientes devem ser educados em relação à doença durante as consultas médicas e, sempre que possível, em grupos de assistência multiprofissional (7).

A seguir serão abordadas as principais classes de medicamentos anti-hipertensivos e seus mecanismos de ação.

1. Bloqueadores adrenérgicos: são drogas que intervêm na transmissão simpática. A maioria dos antagonistas de receptores adrenérgicos é seletiva para os receptores $\alpha$ ou $\beta$, e muitos também são seletivos para seus subtipos (9).

Os antagonistas dos receptores $\beta$-adrenérgicos constituem um grupo importante de fármacos. Foram descobertos em 1958, dez anos após Ahlquist ter postulado a existência dos receptores $\beta$-adrenérgicos. Avanços nessa área conduziram ao propranolol, que é um antagonista potente e bloqueia igualmente os receptores $\beta_{1}$ e $\beta_{2}$ (9).

Os mecanismos de ação dessa classe de drogas anti-hipertensiva são múltiplos, incluindo: diminuição do débito cardíaco, efeitos centrais, readaptação dos barorreceptores, diminuição da liberação de renina e inibição simpática periférica (10).

Como efeito adicional, éimportante ressaltar suas propriedades antiarrítmicas e antianginosas, frequentemente úteis em pacientes hipertensos com comorbidades (10). Podemos citar como exemplos o atenolol, o doxazosina e propranolol (9).

2. Bloqueadores dos canais de cálcio: os antagonistas do cálcio terapeuticamente importantes atuam sobre os canais do tipo L, compreendendo, assim, três classes quimicamente distintas: fenilalquilaminas (verapamil), benzotiazepinas (diltiazem) e diidropiridinas (nifedipina, amlodipina) (9).

Os fármacos de cada uma dessas três classes ligam-se às subunidades $\alpha_{1}$ do canal de cálcio cardíaco do tipo L, mas em locais distintos e que vão interagir alostericamente entre si e com o maquinário de controle da passagem de cálcio, impedindo assim sua abertura e, consequentemente, reduzindo a entrada de cálcio (9).

$\mathrm{Na}$ musculatura lisa causam dilatação arterial/arteriolar generalizada e diminuição de sua resistência, reduzindo dessa forma a pressão arterial $(9,11)$.

A nifedipina para uso sublingual, apesar de sua popularidade nas unidades de emergência de todo o Brasil, inclusive em serviços ligados a escolas médicas, tem sofrido críticas severas pelos especialistas nacionais e internacionais, pois a queda da pressão arterial pode ocorrer de forma abrupta e inesperada, determinando hipofluxo em órgãos nobres, com desastrosas consequências (10).

3. Diuréticos: é considerada a classe de fármacos anti-hipertensivos mais utilizada, em virtude da sua eficácia terapêutica e do seu baixo custo (12). São substâncias com uma ação sobre os rins, atuando de forma a aumentar a taxa do débito 
e volume urinário, consequentemente a excreção urinária de solutos, em especial o sódio e cloreto $(9,11)$.

Seu efeito primário consiste em diminuir a reabsorção de sódio pelos túbulos, causando natriurese (maior débito de sódio), o que por sua vez causa diurese (maior débito de água), sendo o aumento da perda de água secundário à excreção aumentada de sódio, já que o sódio remanescente nos túbulos age de forma osmótica, diminuindo a reabsorção de água $(3,9)$.

O uso clínico mais comum dos diuréticos é para reduzir o volume de líquido extracelular, especialmente em doenças associadas a edema e hipertensão, inibindo a reabsorção tubular em locais diferentes ao longo do néfron renal $(3,12,13)$.

As classes gerais de diuréticos e seus mecanismos de ação são listados a seguir.

3.1 Diuréticos de Alça: a furosemida, ácido etacrínico e bumetanida são diuréticos potentes que diminuem a reabsorção ativa no segmento ascendente espesso da alça de Henle ao bloquear o cotransportador de 1 sódio, 2 cloretos, 1 potássio, localizado na membrana luminal das células epiteliais (Figura 1). Esses diuréticos estão entre os mais potentes usados clinicamente $(3,11)$.

Ao bloquear o cotransporte ativo de sódio, cloreto e potássio na membrana luminal da alça de Henle, os diuréticos de alça aumentam o débito urinário desses eletrólitos e também de outros, bem como o de água $(3,11)$.

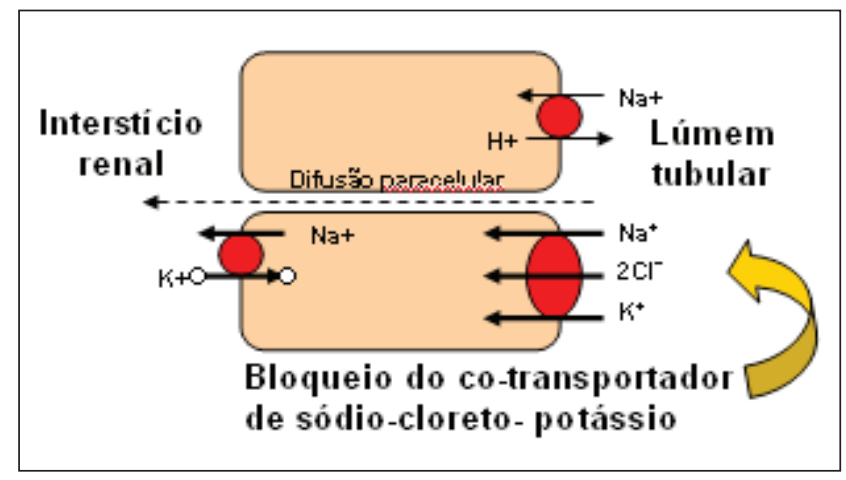

FIGURA 1 - Nesse caso, é possível observar o sítio de ligação dos diuréticos de alça nas células tubulares bloqueando o cotransportador de sódio-cloreto-potássio, favorecendo o débito urinário desses eletrólitos com diminuição da reabsorção de liquido

Fonte: $\mathrm{O}$ autor.
Esse mecanismo pode ocorrer por duas razões: primeiramente eles aumentam muito as quantidades de solutos liberados para as partes distais dos néfrons, atuando como agentes osmóticos e evitando a reabsorção de água, ou interrompendo o sistema multiplicador de contracorrente, diminuindo a absorção de íons da alça de Henle para o interstício medular, reduzindo assim a osmolaridade do líquido intersticial medular, o que compromete a capacidade dos rins de concentrar ou diluir a urina (3).

A baixa osmolaridade do líquido intersticial da medula renal diminui a absorção de água pela alça descendente de Henle, o que produz um débito urinário 25 vezes acima do normal (3). Os diuréticos de alça podem ser eficazes, mesmo em pacientes com a função renal seriamente comprometida (11).

3.2 Diuréticos Tiazídicos: os diuréticos tiazídicos foram os primeiros anti-hipertensivos disponíveis para uso em larga escala. Lançados em meados dos anos 50, continuam a ser administrados, isolados ou em associação, a milhões de hipertensos em todo o mundo. Foi com essa classe de drogas que se demonstrou redução da morbimortalidade com o tratamento anti-hipertensivo (14).

Os derivados tiazídicos, como a clortalidona e hidroclorotiazida, atuam basicamente na parte proximal dos túbulos contorcidos distais, bloqueando o cotransportador de sódio-cloreto na membrana luminal das células tubulares (Figura 2) (3, 12). Sob condições favoráveis, esses agentes fazem com que de $5 \%$ a $10 \%$ do filtrado glomerular passe para a urina (3).

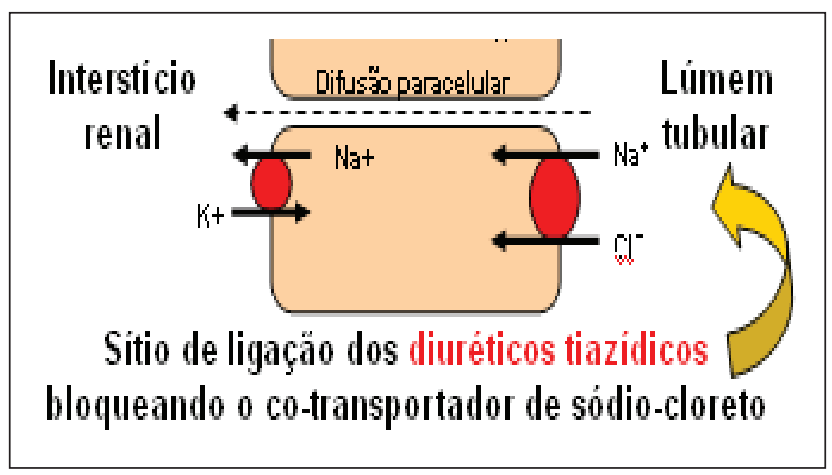

FIGURA 2 - Nessa ilustração é observado o sítio de ligação dos derivados tiazídicos bloqueando o cotransportador de sódio-cloreto, favorecendo o débito urinário desses eletrólitos com diminuição da reabsorção do filtrado

Fonte: $\mathrm{O}$ autor. 


\subsection{Inibidores competitivos da aldos-} terona: temos como antagonistas da aldosterona a espironolactona e esplerenona, que competem com esse hormônio pelos sítios receptores nas células epiteliais do túbulo coletor cortical, reduzindo a absorção de sódio e secreção de potássio nesse segmento tubular (Figura 3). Consequentemente, o sódio permanece no túbulo agindo como diurético osmótico e causando aumento da excreção de água e sódio. Na medida em que esses fármacos bloqueiam os efeitos da aldosterona de promover a secreção de potássio pelos túbulos, eles diminuem a excreção desse íon, aumentando sua concentração no líquido extracelular, e por isso são referidos como diuréticos poupadores de potássio (3).

\subsection{Diuréticos que bloqueiam canais} de sódio nos túbulos coletores: a amilorida é um exemplo de fármaco que inibe a reabsorção de sódio e secreção de potássio de modo semelhante à espironolactona. Entretanto, no nível celular agem diretamente, bloqueando os canais de sódio da membrana luminal das células epiteliais do túbulo coletor (Figura 3). Devido a essa menor entrada de sódio nas células epiteliais do túbulo coletor, ocorre também um menor transporte desse íon pelas membranas basolaterais e, consequentemente, menoratividade da atepase $\mathrm{Na} / \mathrm{K}$. Essa diminuição de atividade reduz o transporte de potássio para as células e, por fim, diminui a secreção de potássio para o líquido tubular. Por essa razão, os bloqueadores dos canais de sódio são também considerados diuréticos poupadores de potássio (3).

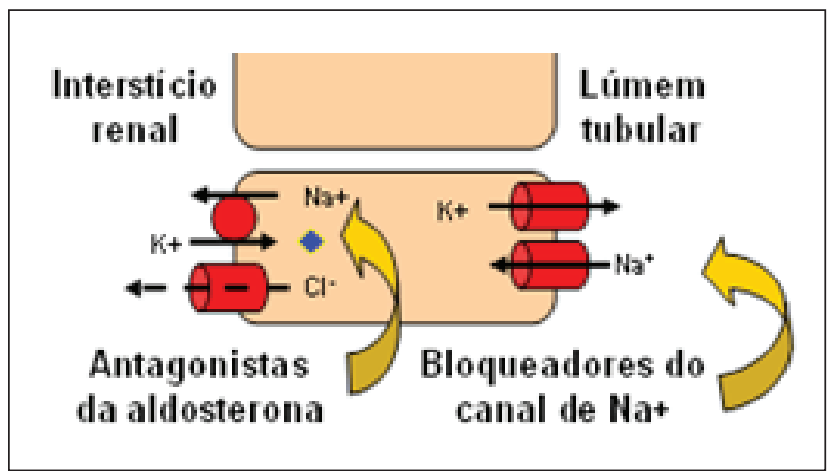

FIGURA 3 - Na região basolateral celular é demonstrada a ação dos antagonistas da aldosterona competindo pelo sítio de ligação (azul) desse hormônio com redução da absorção de sódio e secreção de potássio, e na região apical a ação de bloqueadores diretos dos canais de sódio com função semelhante

Fonte: $\mathrm{O}$ autor.
4. Drogas que intervêm no sistema renina-angiotensina: $\mathrm{O}$ sistema renina-angiotensina aldosterona (SRAA) desempenha importante função na regulação da pressão arterial e da homeostase eletrolítica. Há quase 20 anos, essas substâncias desempenharam relevante papel na terapia da hipertensão arterial e nas doenças cardiovasculares (15).

Essa classe de fármacos apresentou grande avanço desde que o primeiro composto, o captopril, foi liberado para uso clínico em 1981. Atualmente, existem no mercado em torno de 20 inibidores da enzima conversora de angiotensina (ECA), usados em vários países (15).

Os inibidores da ECA impedem a conversão de angiotensina I em angiotensina II (Figura 4), que é um vasoconstritor potente, atenuando assim seus efeitos $(9,15)$.

O uso desse inibidor é a melhor alternativa de medicação por via oral ou sublingual para o tratamento de crises hipertensivas, especialmente aquelas em que o bloqueio do SRAA tenha indicação preferencial, como na insuficiência cardíaca congestiva, AVC, hipertensão arterial maligna e infarto agudo do miocárdio (10).

Essa classe de anti-hipertensivo é formalmente contraindicada na gravidez e em estenose bilateral de artérias renais. Deve ter uso cauteloso em insuficiência renal, estados hipovolêmicos e em hipercalemia $(9,10)$.

Exemplos: enalapril, lisinopril, captopril.

Dentro dessa classe incluem também os antagonistas dos receptores $\mathrm{AT}_{1}$ de angiotensina II, por exemplo, o losartan (16). Sabe-se que a angiotensina II liga-se a dois subtipos de receptores, $\mathrm{AT}_{1}$ e o $\mathrm{AT}_{2}$, e todas as funções conhecidas da angiotensina II se dão pela ligação com os receptores $\mathrm{AT}_{1}$. Os antagonistas dos receptores da angiotensina II (AII) têm como mecanismo de ação o bloqueio das ações da AII pela ocupação específica do receptor $\mathrm{AT}_{1}$, exercendo consequentemente ação anti-hipertensiva e protetora para os diferentes órgãos-alvo da hipertensão arterial (9)

6. Outros mecanismos: relaxante do músculo liso, através do aumento seletivo da permeabilidade da membrana ao potássio, ocasionando hiperpolarização da membrana, desligando os canais de cálcio voltagem-dependentes e inibindo a geração de um potencial de ação (exemplo: minoxidil). Há também drogas que afetam a síntese da noradrenalina, por ser um precursor de transmissor falso (exemplo: metildopa) (9). 


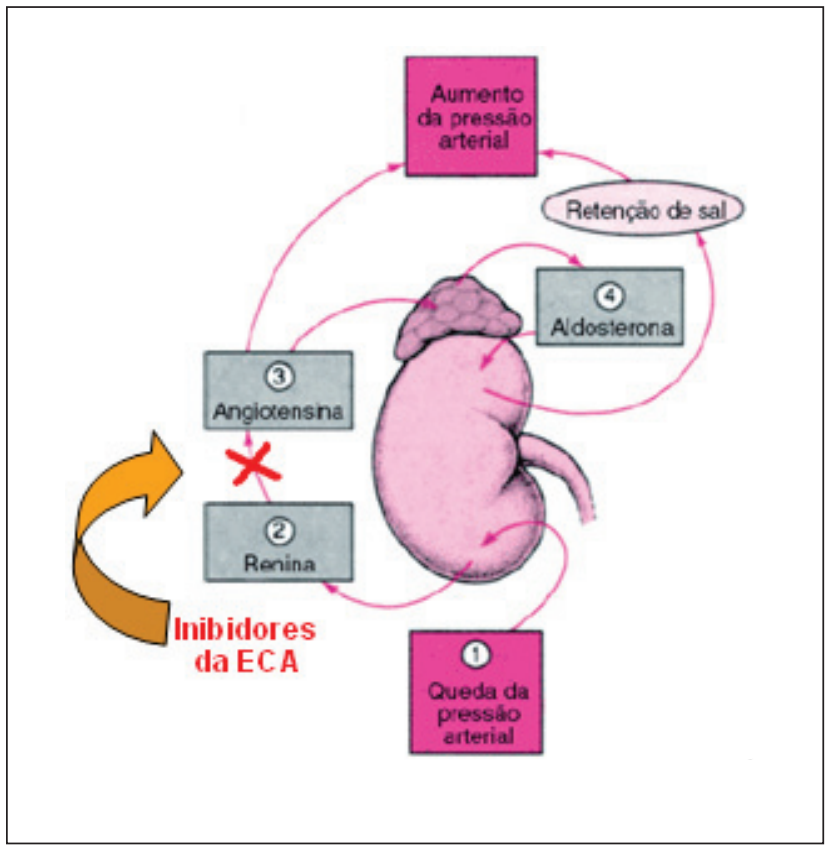

FIGURA 4- Esquema mostrando a ação dos inibidores da enzima conversora de Angiotensina (ECA, ) que impede a conversão de angiotensina I em angiotensina II

Fonte: O Autor.

\section{DISCUSSÃO}

Contrariando o ponto de vista mais antigo, de que a hipertensão arterial fosse "essencial" para manter a vida, reduzir a pressão melhora muito o prognóstico dos pacientes com hipertensão. Controlar a hipertensão que é assintomática sem produzir efeitos colaterais inaceitáveis, portanto, é uma necessidade clínica importante, que, em geral, é bem atendida pelos fármacos modernos.

Ao longo dos anos, a terapia farmacológica anti-hipertensiva mostrou um grande impacto em termos de redução do risco para os pacientes. Para o início do tratamento, em geral se dá preferência para uma droga de primeira linha, iniciando preferencialmente em doses baixas e sob regime de monoterapia ou terapia combinada.

O bloqueio do sistema renina-angiotensina-aldosterona (SRAA) constitui uma excelente opção terapêutica para a hipertensão arterial (HA) bem como para a cárdio e nefroproteção (17).

Rodrigues (10) relata que o uso do captopril (inibidor da enzima de conversão da angiotensina) é a melhor alternativa de medicação por via oral ou sublingual para o tratamento de crises hipertensivas, especialmente aquelas em que o bloqueio do SRAA tenha indicação preferencial, como na insuficiência cardíaca congestiva, AVC, hipertensão arterial maligna e infarto agudo do miocárdio.

Segundo Santello e Mion Jr. (14), a associação de captopril à hidroclorotiazida, um diurético tiazídico em dose baixa, oferece vantagens características do anti-hipertensivo ideal, como controle da pressão arterial (PA), redução da mortalidade cardiovascular, proteção cardíaca e renal, custo acessível e baixa incidência de efeitos colaterais.

Apesar do captopril ser um medicamento frequentemente prescrito nas unidades de saúde, o enalapril apresenta-se uma droga consideravelmente mais potente, e segundo Goodman e Gilmam (6), um ponto interessante é seu tempo de ação ser mais prolongado, sendo muito eficazes tanto em monoterapia como em associação com outras drogas, especialmente diuréticos.

Os antagonistas dos receptores $\beta$-adrenérgicos são menos tolerados que os IECAs ou antagonistas de AT1, e as evidencias que sustentam seu uso de rotina são menos fortes que para outras classes de anti-hipertensivos. São úteis para hipertensos com alguma indicação adicional para um bloqueio $\beta$, como angina ou insuficiência cardíaca (9)

De acordo com Perroti et al. (7), os diuréticos tiazídicos são os fármacos de primeira escolha nos idosos, exceto naqueles casos em que há uma indicação preferencial. Apesar de alguns autores sugerirem que a clortalidona e a hidroclorotiazida (diuréticos tiazídicos) serem equivalentes, propriedades farmacológicas e alguns estudos mostraram que a clortalidona parece ser mais potente que a hidroclorotiazida (12).

Mesmo em baixas doses, os tiazídicos mantêm sua eficácia anti-hipertensiva, com baixo risco de efeitos colaterais (como hipopotassemia, hipomagnesemia e hiperuricemia), e apresentam baixo custo e benefícios cardiovasculares comprovados (7).

Apesar das evidências em favor da clortalidona, a hidroclorotiazida continua sendo o diurético tiazídico mais comum utilizado na prática clínica. Essa diferença ocorre principalmente em relação ao reduzido número de combinações fixas contendo clortalidona, comparado com a grande disponibilidade de combinações fixas contendo hidroclorotiazida.

No entanto, o médico deve considerar as diferenças clínicas e farmacológicas ao escolher o diurético tiazídico mais apropriado (12). 
Além do tratamento farmacológico das HAS, deve-se também envolver medidas não farmacológicas, como o aumento dos exercícios físicos, redução do sal das dietas e das gorduras saturadas com aumento de frutas e fibras, redução do peso corporal e do consumo de álcool e tabagismo.

\section{CONCLUSÃO}

A hipertensão arterial continua sendo uma das mais importantes morbidades nos pacientes adultos, estando direta ou indiretamente relacionada à principal causa de morte no País. O idoso em especial apresenta particularidades que devem ser avaliadas no momento em que é decidido pelo tratamento medicamentoso da hipertensão arterial, por conta de alterações fisiológicas próprias do envelhecimento. $\mathrm{O}$ esquema de tratamento antihipertensivo deve manter a qualidade de vida do paciente, de modo a estimular a adesão às recomendações prescritas seguindo a lógica de não combinar medicamentos com mecanismos de ação similares. O tratamento medicamentoso da HAS deve ser associado ao não medicamentoso, havendo dessa forma a necessidade de um trabalho multiprofissional no sentido de reduzir a pressão arterial. A HAS é considerada uma patologia multifatorial com alta prevalência na população, e por isso um diagnóstico correto e tratamento apropriado podem melhorar consideravelmente a sobrevida desses pacientes, declinando assim os índices de mortalidade em comparação com os números que vêm sendo observado. Assim, frente à variedade de classes medicamentosas disponíveis, muitas com longa duração de ação, e sempre considerando as características individuais dos pacientes, é possível se obter controle da pressão arterial na maioria dos casos, quer com monoterapia ou com associação de drogas, com benefícios que vão além do controle pressórico, como a proteção de órgãos-alvo da doença hipertensiva, e melhora na adesão ao tratamento.

\section{REFERÊNCIAS}

1. Pellizzaro MC, Pancheniak EFR. Assistência farmacêutica no tratamento de doenças cardiovasculares e hipertensão. Infarma. 2003;15(9-10):2003.
2. Marte AP, Santos RD. Bases fisiopatológicas da dislipidemia e hipertensão arterial. Revista Brasileira Hipertensão. 2007;14(4):252-7.

3. Guyton AC, Hall JE. Tratado de Fisiologia Médica. 11a ed. Rio de Janeiro: Elsevier; 2006.

4. Pimenta E, Calhoun DA. Uso dos antagonistas da aldosterona no tratamento da hipertensão arterial refratária. Revista Brasileira Hipertensão. 2007;14(3):180181.

5. Page C P. Farmacologia integrada. São Paulo: Manole; 1999.

6. Goodman LS, Gilman A. As bases farmacológicas da terapêutica. 8a ed. Rio de Janeiro: Guanabara Koogan; 1991.

7. Perrotti TC, Campos Filho J, Uehara CA, Almada Filho CM, Miranda RD. Tratamento farmacológico da hipertensão no idoso. Revista Brasileira Hipertensão. 2007;14(1):37-41.

8. Rosa RF, Franken RA. Fisiopatologia e diagnóstico da hipertensão arterial no idoso: papel da monitorização ambulatorial da pressão arterial e da monitorização residencial da pressão arterial. Revista Brasileira Hipertensão. 2007;14(1):21-4.

9. Rang HP, Dale MM, Ritter JM, Flower RJ. Rang \& Dale farmacologia. 6a ed. Rio de Janeiro: Elsevier, 2007.

10. Rodrigues CIS. Tratamento das emergências hipertensivas. Revista Brasileira Hipertensão. 2002;9:353-8.

11. Nigro D, Fortes ZB. Efeitos farmacológicos dos diuréticos e dos bloqueadores dos canais de cálcio. Revista Brasileira de Hipertensão. 2005;12(2):103-7.

12. Pimenta E. Hidroclorotiazida x clortalidona: os diuréticos tiazídicos são todos iguais? Revista Brasileira Hipertensão. 2008;15(3):166-7.

13. Henry JB. Diagnósticos clínicos \& tratamento por métodos laboratoriais. 18a ed. São Paulo: Manole, 1995.

14. Santello JL, Mion Jr D. Captopril associado à hidroclorotiazida no tratamento da hipertensão leve e moderada. Arquivo Brasileiro de Cardiologia. 1998;71(5):713-6.

15. Ribeiro JM, Florêncio LP. Bloqueio farmacológico do sistema renina-angiotensina-aldosterona: inibição da enzima de conversão e antagonismo do receptor AT1. Revista Brasileira de Hipertensão. 2000;7(3):293-302. 
16. Bicket DP. Using ACE inhibitors appropriately. American Family Physician. 2002;66(3):461.

17. Baumgratz PR. Duplo bloqueio do sistema renina-angiotensina-aldosterona: indicações clínicas e abordagem da hiperpotassemia e do aumento da creatinina. Hipertensão. 2007;10(4):152-7.

18. Manual Merck para a família. [acesso 14 dez. 2009]. Disponível em: http://www.manualmerck.net/images/thumbnail/p_116.gif.

Recebido: 08/07/2007

Received: 07/08/2007

Aprovado: $28 / 11 / 2007$

Approved:11/28/2007 